\title{
A review on orchestration distributed systems for IoT smart services in fog computing
}

Nor Syazwani Mohd Pakhrudin, Murizah Kassim, Azlina Idris

Faculty of Electrical Engineering, Universiti Teknologi MARA, Malaysia

\begin{tabular}{|c|c|}
\hline Article Info & ABSTRACT \\
\hline Article history: & This paper provides a review of orchestration distributed systems for IoT \\
\hline Received Mar 30, 2020 & $\begin{array}{l}\text { smart services in fog computing. The cloud infrastructure alone cannot } \\
\text { handle the flow of information with the abundance of data devices and }\end{array}$ \\
\hline Revised Jul 28, 2020 & interactions. Thus, fog computing becomes a new paradigm to overcome the \\
\hline Accepted Aug 6, 2020 & $\begin{array}{l}\text { problem. One of the first challenges was to build the orchestration systems to } \\
\text { activate the clouds and to execute tasks throughout the whole system that has }\end{array}$ \\
\hline Keywords: & $\begin{array}{l}\text { to be considered to the situation in the large scale of geographical distance, } \\
\text { heterogeneity and low latency to support the limitation of cloud computing. }\end{array}$ \\
\hline Distributed systems & Some problems exist for orchestration distributed in fog computing are to \\
\hline Fog computing & $\begin{array}{l}\text { fulfil with high reliability and low-delay requirements in the loT applications } \\
\text { system and to form a larger computer network like a fog network, at different }\end{array}$ \\
\hline Internet of things & geographic sites. This paper reviewed approximately 68 articles on \\
\hline Orchestration & orchestration distributed system for fog computing. The result shows the \\
\hline Smart services & $\begin{array}{l}\text { computing that have been compared in terms of Borg, Kubernetes, Swarm, } \\
\text { Mesos, Aurora, heterogeneity, QoS management, scalability, mobility, } \\
\text { federation, and interoperability. The significance of this study is to support } \\
\text { the researcher in developing orchestration distributed systems for IoT smart } \\
\text { services in fog computing focus on IR4.0 national agenda. }\end{array}$ \\
\hline
\end{tabular}

This is an open access article under the CC BY-SA license.

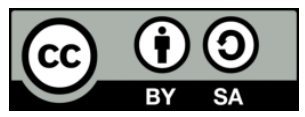

Corresponding Author:

Murizah Kassim

Faculty of Electrical Engineering

Universiti Teknologi MARA

40450 Shah Alam, Selangor, Malaysia

Email: murizah@uitm.edu.my

\section{INTRODUCTION}

In recent years, the rapid development of distributed computing requires a decentralized computing system to encounter the varying difficulties of different IoT applications like QoS, latency, privacy and scalability. Due to the increased data speed and volume, it may not be possible or even unachievable to transfer big data from IoT devices into the cloud due to bandwidth constraints in some cases [1]. The importance of the cloud as an interface for the integration of distributed heterogeneous computer resources and the availability of integrated and seamless computing systems to end-users as services has gradually increased in recent years in the handling of the actual handing out of significant numbers of heterogeneous wireless IoT devices data [2]. Conventional cloud computing architecture is unsuited to simultaneously support trillions of IoT devices at the network edge in real-time, because of certain shortcomings [3]. Cloud computing is didn't intend for the large scale of geographical distance, heterogeneity and low latency, which was most relevant during the fourth industrial revolution, in Industry 4.0 and IoT related cases [4]. A new computing model is therefore needed to restrict cloud-based computing to satisfy the needs of these critical IoT applications for latency. New technologies focused on distributed IoT devices will face their difficulties. 
Each IoT system can process data on its own or send it to a processing server. A distributed storage system must fulfil several criteria for use in the fog computing environment. Fog computing is the paradigm between cloud and endpoint devices that brings processing, storage and networking resources close to the end devices [5]. It should be clearly stated that fog computing does not substitute for cloud computing but rather complements these two technologies. Fog computing expands cloud computational systems to the edge of the network where data and applications exist between the data source and the cloud to address delaying sensitive IoT devices in a cloud environment.

Big data are very various data and are necessary to store, maintain and process the data within a proper time frame even from the popular IoT or any other paradigm hardware and software environment. The cloud services provide user-friendly applications and tools for cloud services offered by public, private, and certain approved entities that are centralized, secure and cost-effectively. As for the cloud, storage and infrastructure are at the edge of the network. Fog computing is capable of handling knowledge streaming on the edge of the network created by the Internet of things. The development of these services at the edge of the fog computing system will lead to new business models and network operators' opportunity [6]. Fog provides end-users in data, storage computing, and application services. Its proximity to end-users, its dense geographical distribution and its mobility support are characteristic features of Fog. Services are hosted on the edge of a network or even on end devices like set-top boxes or access points. It will allow the IoT to solve issues such as reduced service latency and increased QoS. The fog system is suited for real-time big-data and real-time analysis due to its wide geographic distribution. Fog supports densely distributed data collection points and thus improves the frequently mentioned dimensions of big data such as volume variety and speed with a fourth axis.

Internet of things (IoT) defined as a device for receiving or transmitting data via transceivers, such as wires, vehicles, actuators, smart grids, or any smart devices so that devices are connected [7]. The growth of new-generation information technology, including cloud computing (CC), IoT, big data analytics (BDA), artificial intelligence, Cyber-physical system (CPS), and other technology, leads towards a rise in smart device consumer creation in 2025 [8]. Cloud computing is needed to deal with the explosion data that extended to be accessible through the internet [9]. Cloud computing is internet-based and allows shared computer resources such as storage, applications, data management, apps, etc. The cloud computer offers software, interface, network, storage, security data, etc. The IoT will produce unanticipated quantities and varieties of data. The cloud infrastructure alone cannot handle the flow of information with the abundance of data, devices and interactions. As the cloud provides easy and cheap access to, save, and even network, these centralized services can lead to delays and performance problems on devices and data far away from a central public cloud or data centre source. Fog computing can help to overcome these limitations by resolving the total bandwidth, latency and inefficient QoS. Fog computing expands the cloud to devices generating IoT data and acting on it. Fog computing includes data processing or analysis elements in the distributed cloud and edge devices. Figure 1 shows the three layers for the architecture of fog computing [10]. The layer that closest to the end customer and physical condition is called edge layer. Edge layer contains many types of IoT devices such as sensor, mobile phone and so on. The framework of the edge was arranged to the fog layer. Fog layer generally including switches, gateway, base stations and others.

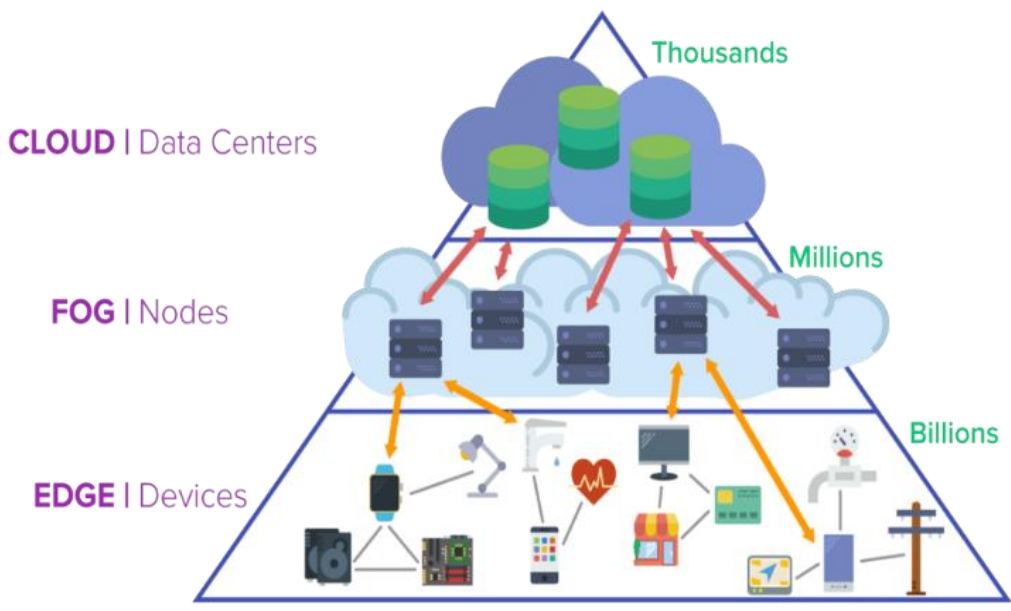

Figure 1. The hierarchy of layers in fog computing 
Figure 2 is representing a fog computing architecture for the whole IoT system [11]. The end devices such as sensor and actuators of IoT devices located in at the bottom layer in the fog architecture. The sensor will stream the data to the network along with the application that enhances their functionality. The next layer's for fog computing is the network. This network engaged communication with the edge device such as gateways. At this stage, components for resource management act as monitoring services to track the state of available to process incoming tasks. The usage of APIs to build complex functionalities become the next stage for fog computing. Finally, the application layer is to deliver the application services to the users or clients.

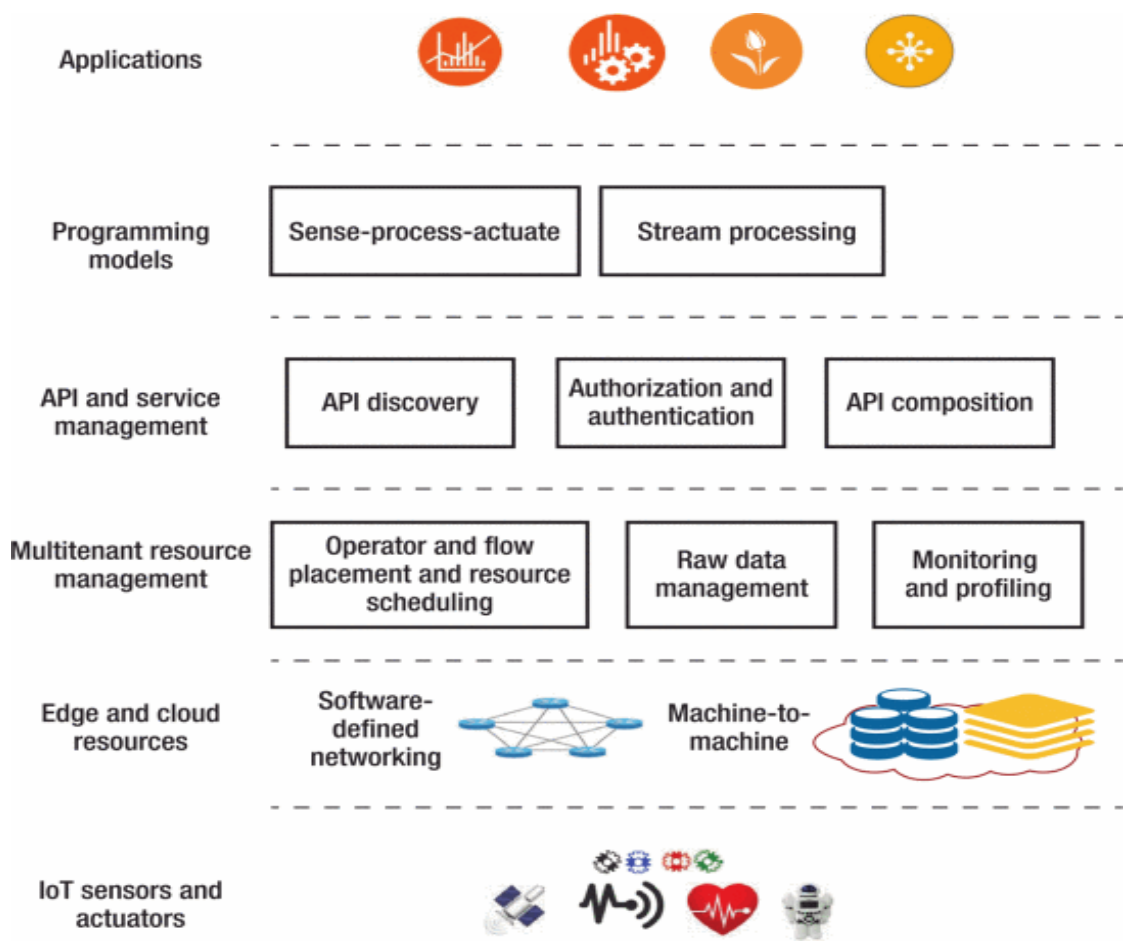

Figure 2. Fog computing architecture

Cloud platform orchestration systems are responsible for creating, maintaining, and allocating device and bandwidth resources for the services requested. Conventional methods in data centres, where they are a single fault point and potentially a value container, rely on centralized solutions [12]. Orchestration means an automated centralized process, which manages the interaction of applications or services. Orchestration also uses a structured approach to the design of programs and facilities. While other scenarios such as the cloud have been subject to various orchestration methods, the fog has unique characteristics, such as the distributional existence, the complexity of its devices and resource constraints [13]. Orchestration is a crucial term of distributed organization, heterogeneous systems and facilities administration. The method of orchestration was implementing in both the cloud-based IoT and fog-based IoT situations. Due to the nature of the fog measurement, such as heterogeneous distributed devices and their limiting resources, the function of orchestration is easily justifiable for the case of IoT fog. An orchestration helps to adapt many specific fog computing techniques, limitations and applications [14]. Networking technology enables the smooth communication between a wide range of devices including cell phones, tablets, TVs, servers, microphones, lighting and other appliances. By selecting the correct data traffic load from WSN, a reduction in the chance to maintain QoS can be minimized [15].

\section{ORCHESTRATION CHALLENGES FOR IoT APPLICATION}

Fog computing brings challenges at many different levels. By looking from a broader perspective, one of the first challenging issues is the modelling of the orchestration element that needs to be able to perform the deployment of the cloudlets and handle tasks inside the environment. The combination of IoT, fog and cloud embrace a complex scenario wherein some case it is not suitable to migrate or apply well- 
known solutions or mechanisms from other domains or paradigms. The existing IoT applications are very diverse in terms of reliability, scalability and security. The complexity of Fog nodes is a big challenge in terms of location, setup and the features of the fog node increases this complexity significantly. It presents a fascinating research challenge, namely how the method of evaluating and choosing the right IoT and fog components for an application framework can be optimized while meeting non-functional requirements like stability, network latency, QoS, etc. The transition from cloud to fog raises many critical obstacles. It requires the need to support on-orchestration and reliable fog services that are critical to the success of the future IoE, and emerging IoT [16]. The fog computing challenges is how to customized and pick the right IoT devices and fog components to create an application workflow while fulfilling non-functional needs such as protection, network latency and QoS. A realization of dynamic graph generation and partitioning during the runtime to adapt possible solutions from the scale and dynamic of the internet of items remains an unresolved problem [17]. Several other open challenges and areas of research include safety factors such as fog node authentication, rogue node detection, privacy, intrusion detection system, access control, and data protection and dynamic nature of fog nodes [18].

\section{MOTIVATION}

Seeing from a bigger perspective, the development of the orchestration systems which should be able to implement the clouds and perform tasks throughout the system one of the first challenges. The main's motivation in fog computing is latency. Ultra-reliable low-latency in 5G defines determines the efficiency of a PER around 10-6 and the transmission time of end-to-end as low as $1 \mathrm{~ms}$ that many IoT applications will fulfil with high reliability and low-delay requirements [19]. Mobile networks cannot be suitable in terms of technology and management for some critical situations, such as industrial control and manufacture. In November 2019, the average transatlantic round time between London and New York in the Verizon enterprise network was 70.439 [20]. The perfect orchestration of the tasks in the fog nodes is necessary to fulfil these strict conditions. In fog computation, the second motivation is large-scale and distributed. Fog nodes may belong to separate parties, which form a bigger computer network like a fog network, at different geographic sites. The architecture supporting fog computing must be scalable and take into consideration the specific preferences and safety concerns of the owners of fog infrastructure. A dynamic and autonomous is the third motivation for this work. As a result of the on-setting of IoT applications and the mobility of fog nodes, the condition of a fog network is changing dynamically, and some fog nodes are unconnected with their networks. For order to tackle complexities, fog computation should be independent.

Besides that, the quality-of-Service become one of the research motivations. IoT implementations will have its specifications for the quality of service (QoS) in the affinity-ware offloading phase to be met, including delays, performance levels such as streaming rates for video applications and the data locality. Therefore, it is not easy to decide how several applications should be deployed simultaneously in the shared fog network. Bandwidth is also important's for this motivation research and serving requests at the network's edge would save the bandwidth between edge and core. Saving bandwidth would not just reduce costs but also reduce the amount of $\mathrm{CO} 2$ emissions from the network devices. In 2018, ICT contributes to 6-10\% of global electricity consumption, or $4 \%$ of greenhouse emissions, and this figure rises annually between $5 \%$ and $7 \%$ [21]. A route usually works at $60 \%$ of its capacity. Even though it's inactive, they use almost as much energy and don't allow switching off during off-peak hours, as when they are operating at their full capacity.

\subsection{Reviews structure}

The most common way to look for review structure is through the online search functions of popular's publishing databases. Figure 3 shows the review structure for the orchestration distributed systems for IoT smart services in fog computing. Most of the selected journals and proceedings from IEEE Xplore database which index in SCOPUS and science direct has been chosen by those reviews to search for candidates of literature reviews. Google Scholar has also been used by because it provides all sorts of papers covering peer reviews of items from selected databases. The search keywords were defined based on the research structure. The main factors for the following search query are orchestration distributed systems, IoT smart services, fog computing, IoT applications, Edge and Cloud computing and IoT communications. For each review paper, it needs to contain the keywords of the smart services (such as smart healthcare, smart city, smart wearables, and so on), the architectures orchestration distribution, fog computing motivations and criteria of fog computing. Total reviews papers are about 68 which 40 journals and 27 proceedings.

\subsubsection{Study of reviews areas}

All the review paper was select from 5 years back started from 2015 until 2020. Most of the journal and proceedings have been select from 2018 until 2020. Only a few journals and proceedings were selected between from the year 2015 until 2017.

A review on orchestration distributed systems for IoT smart services in ... (Nor Syazwani Mohd Pakhrudin) 


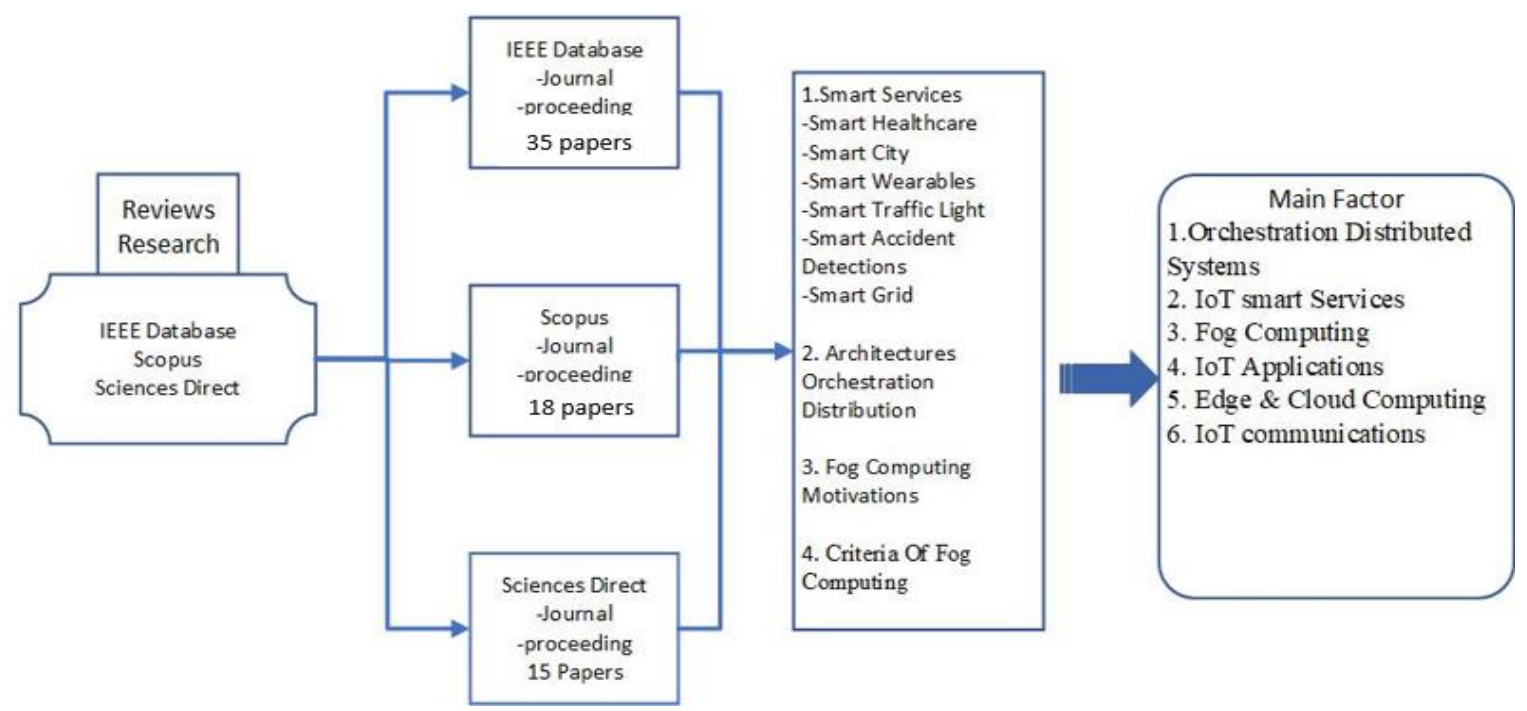

Figure 3. The review structure for the orchestration distributed systems for IoT smart services in fog computing

\section{IoT APPLICATIONS REVIEWS}

\subsection{IoT smart services applications}

The number of wearable computing devices is almost unstoppable, such as smartwatches, smart devices (smart metres, smart cities or smart cars), large-scale wireless sensor networks, and the Internet of Things (IoT) is almost constant. Fog computing offers a range of theoretically competitive benefits over cloud storage, including in-house processing, data store privacy and analysis, a distributed and federated near-by computer connectivity, and emerging business possibilities for accelerated innovation. The thorough use of both paradigms in conjunction created unprecedented opportunities to create's new technologies and ideas that were previously unthinkable. Smart's service is a digital service that interacts with knowledge gathered and analysed by networking, smart's technologies and platforms. In comparison to industry 4.0 technologies, smart networks require cross-functional industrial, which can only happen in one specific field $[22]$.

\subsubsection{Smart traffic lights}

A smart traffic light system (TLS) allows an STL used to at every intersection. The STL was fitted by sensors that sense the movement of pedestrians and bikes crossing the road and measure the distance and speed of the vehicles arriving from either side. The STL even speeds down the signal for red-crossing traffic and also switches its process to prevent accidents. The TLS has three primary objectives: avoiding collisions, ensuring smooth traffic, gathering the necessary data to assess and develop the network [23].

\subsubsection{Smart wearables}

In the market today are trendy smart devices, like smartphones, tablets, PCs, netbooks, etc. These machines are small and portable, making them functional [24]. Wearable technology is an electronic system of some type intended for use in the user's body. The word wearable computing means computation or networking capability, but wearables may differ in fact. The apps are hands-free phones, powered by microprocessors and able to send and receive data on the Internet. Wearable hardware was developed based on the development of mobile networks. The production of wearable technologies now appears to concentrate on more advanced and realistic uses, including consumer accessories. The use of microchip implants now removes keys and passwords. Embedded based on fingertip recognition, the chips are similar to those used to track missing pets using near-field communication (NFC) or radio frequency identification (RFID). The volume of data aggregated and combined with the cloud is growing with the growing use of wireless and portable sensory network systems [25].

The medical data is collected by the intelligent edge devices such as wearables, wrist-bands, smartwatches, smart textiles etc. The intelligence refers to knowledge of analytics, devices, clinical application and the consumer behavior. Such smart data is structured, homogeneous and meaningful with negligible amount of noise and meta-data [1]. The big data and quiet recently smart data trend had revolutionized the biomedical and healthcare domain. With increasing use of wireless and wearable body 
sensor networks (BSNs), the amount of data aggregated by edge devices and synced to the cloud is growing at enormous rate.

\subsubsection{Smart accident detection}

Every year a significant number of deaths occur worldwide due to prolonged rescue delays. To order to detect the efficient and track road accidents, vehicles installed to specialized technologies and roads fitted with modern facilities are necessary. Nevertheless, in less developed nations, these networks and technology automobiles are rare. Therefore, low-cost solutions are compulsory in these countries to tackle the problem. Internet of things (IoT)-based applications has begun to be used to detect and track accidents at the roadside. However, the centralization and remoteness of cloud services can lead to an increase in time, causing serious's concern regarding its effectiveness in emergencies; all delays need to eliminate as far as possible while life-threatening conditions are involved. Fog computing has become a Middleware model for solving the issue of latency, which puts cloud-like services closer to terminals [26]. Because of the distributed design of the infrastructure, a cloud-based incident and emergency recovery program will face problems related to latency and bandwidth. Fog computing, which offers reduced latency, connectivity assistance, improved flexibility and scalability, is a new technology to help solve these problems. Besides, the use of mobile sensors makes for cheaper and faster installation of emergency and response devices in legacy vehicles.

\subsubsection{Smart grid}

Smart grids were built to conserve electricity by using ICT, where a completely integrated power delivery network is available, where both utilities and customers communicate with one another to exchange knowledge. However, it is important's to monitor consumption and generate power to achieve these properties [27]. Data need to be gathered and exchanged to gain real-time connectivity between customers, providers, transmission lines and generators via smart grid topology [27]. Hisham et al. [28] the IT gaming device that improvises the current forms and produces electricity and the Internet of Things (IoT) proposed. This system is programmed to generate energy when playing games by referring to the user scores. The system was built consisting of hardware and system designed with Arduino, PHP programmes that connect via smartphones and web servers. The cloud data on Fusionex Giant is collected. Unity 3D game engine has been creating to enable users to select and play online games. The system results from the control of mobility by generating electricity when playing games on smartphones.

\subsubsection{Smart city}

The smart city model is a product of the convergence of ICT and the IoT network to tackle contemporary urbanization challenges. The development of smart cities includes the whole city structure, as well as infrastructure management. It is built and maintained through integrated technologies, including sensors, electronics and networks [29]. This incorporation aims to make operations and public infrastructure more effective, to enhance the quality of life of the community and to promote environmental protection while ensuring "smart" city control. Nonetheless, smart city technologies vary from smart traffic control, smart house, smart living, smart manufacturing and smart buildings. Smart city applications incorporate advanced IoT technology by linking people in a community so that all consumers have the right information in real-time at the right time [30]. Santos et al. [31] proposed a fog computing framework that allows for independent management and orchestration of $5 \mathrm{G}$ enabled intelligent cities. The findings reveal that, compared with centralised cloud systems, the current architecture substantially reduces network bandwidth consumption and latency.

\subsubsection{Smart healthcare}

IoT is a technology which radically disrupts the ecological health system. The concept of smart health care is changing with the advent of information technology. Smart technology integrates a new wave of IT innovation such as the internet of things, large-scale applications, cloud computing, artificial intelligence to make the entire revolution efficient and sustainable in the health sector [32]. The large quantities of data generated from these sensors and devices have to be used to make informed decisions for caregivers and decision-makers. To do that, it is crucial that IoT data streams are processed in near-real-time and that data analytics tools are used to learn from events in the healthcare facility or current patient health conditions. Time-sensitive applications, such as surveillance cameras processing videos, are unable to tolerate sending cloud streams due to bandwidth and latency requirements of networks [33]. The adoption of ICTs in the healthcare sector has created the smart health concept for promoting the ubiquitous healthcare services of smart cities through the use of the contextual and sensor network [34]. The centralized cloud provider can not offer medical facilities that are closely connected to geographical location since the primary networks are still congested. To control the geographical propagation of illness is cumbersome coordinating the local or physical network with the remote cloud servicer. Enhanced hardware, storage and smart health

A review on orchestration distributed systems for IoT smart services in ... (Nor Syazwani Mohd Pakhrudin) 
centers are needed for the latest, location-related health systems [35]. The internet of things also used a smartphone device for more effective personal tracking. The mobile app will gather information and data from the database and make the user more interactively to track his health [36].

\subsection{Architecture of orchestration distributed in fog computing}

The fog orchestrator is used as a monitoring panel on a workstation or cloud datacenter and based on global knowledge in all operational structures. The key task is to pick services and execute an overall operation configuration focused on network security, stability, and specifications for system performance. It should be noted that the orchestrater can be used as a centralized controller in a distributed and fault-tolerant manner, and without the introduction of one single failure point [37]. Figure 4 shows as design for fog environment for IoT-enable in orchestration distributed systems.

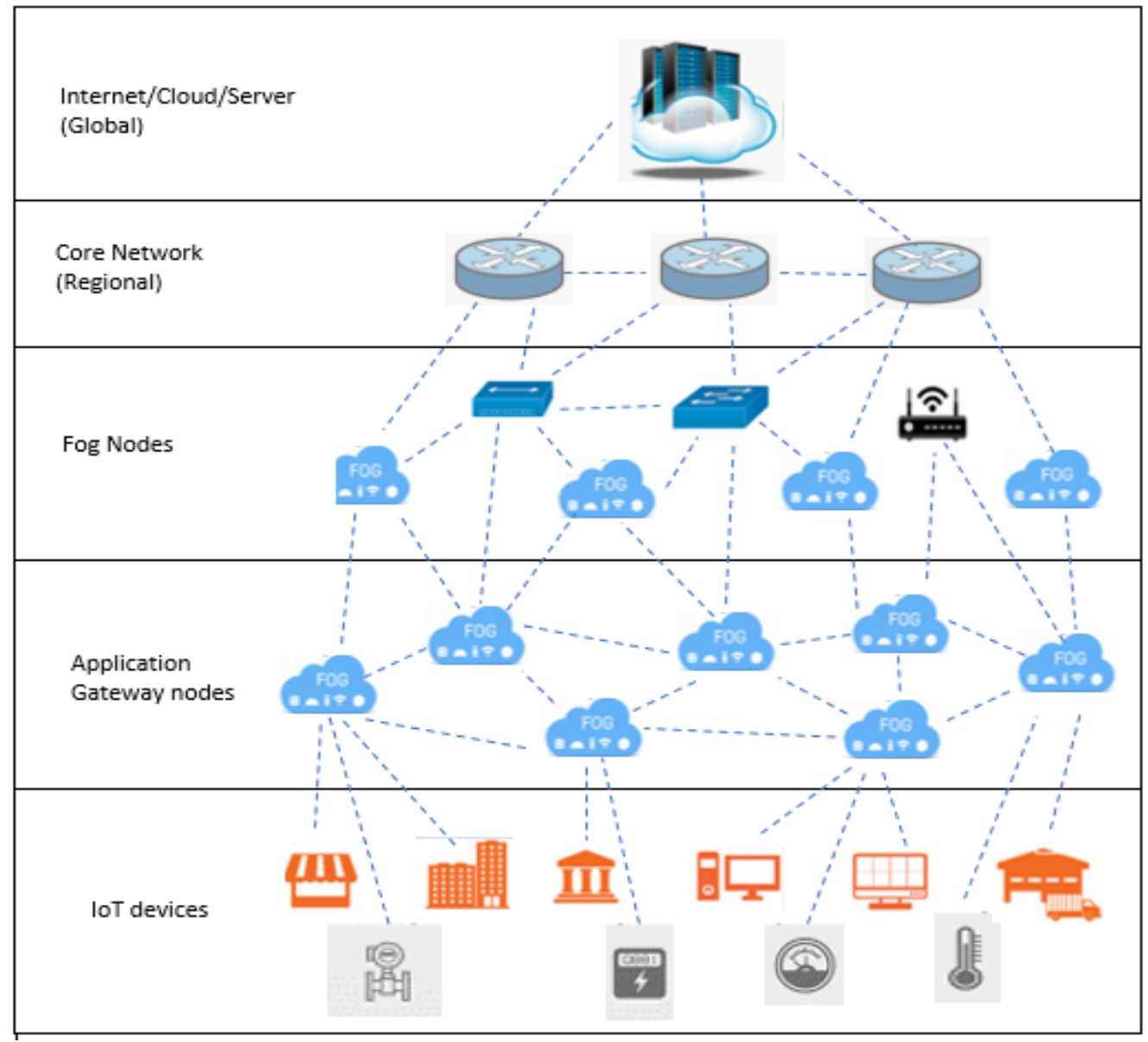

Figure 4. Fog environment for IoT-enable in orchestration distributed systems

\subsection{Reviews gaps}

Reviews gaps between orchestration distribution systems, fog computing and latest IoT smart services is derived in presenting the critical areas towards implementation of this project.

\subsubsection{Review on orchestration distribution system}

The integration of these fog processing devices requires a variety of challenges. At least the analysis programming model has to be distributed. Therefore, to transparently incorporate IoT implementations, the heterogeneous architecture for fog systems of different capacities, hardware and software include virtualization technologies. The status of several IoT apps and fog devices must be managed by a centralized repository in the head office. The system that contain in the orchestration distributed are Borg, Kurbernetes, Swarm, Mesos, and Aurora which are shown in Table 1. 
Table 1. Evaluation system classification for orchestration distribution system

\begin{tabular}{|c|c|c|c|c|c|c|}
\hline System & Scalability & $\begin{array}{c}\text { High } \\
\text { Availability }\end{array}$ & $\begin{array}{c}\text { Application } \\
\text { QoS }\end{array}$ & $\begin{array}{c}\text { High } \\
\text { Utilization }\end{array}$ & $\begin{array}{c}\text { High } \\
\text { Throughput }\end{array}$ & Review paper \\
\hline Borg & $\checkmark$ & $\checkmark$ & $x$ & $\checkmark$ & $\checkmark$ & [38-40] \\
\hline Kubernetes & $\checkmark$ & $x$ & $x$ & $x$ & $x$ & [41-47] \\
\hline Swarm & $\checkmark$ & $\checkmark$ & $x$ & $\checkmark$ & $\checkmark$ & [48-53] \\
\hline Mesos & $\checkmark$ & $\checkmark$ & $x$ & $x$ & $x$ & [54-59] \\
\hline Aurora & $\checkmark$ & $\checkmark$ & $x$ & $x$ & $x$ & {$[60,61]$} \\
\hline
\end{tabular}

\subsubsection{Review on fog computing}

Concerning the studies observed and filtered in the distribution, we find problems that remain open in distributed applications with several levels to handle dependence between containers. Anything like a plane of orchestration may define its elements, its dependency and its life cycle in the container. The short requirements for determining the fog machine environments are considered important. Table 2 shows the criteria of heterogeneity, QoS management, scalability, mobility, federation and interoperability.

Table 2. Evaluation criteria for fog computing

\begin{tabular}{|c|c|}
\hline Criteria & Definition \\
\hline Heterogeneity C1 & $\begin{array}{l}\text { Fog and server nodes in the computing and storage capabilities are very heterogeneous. Heterogeneity } \\
\text { will be able resolved by fog. }\end{array}$ \\
\hline QoS management C2 & $\begin{array}{l}\text { Leading to proximity to IoT apps and end-users, Fog can support applications in real-time. However, } \\
\text { depending on the position of device modules, latency varies significantly and hence involves control of } \\
\text { QoS. }\end{array}$ \\
\hline Scalability C3 & $\begin{array}{l}\text { A wide range of end-user computers, applications, domains and nodes is necessary for Fog. This must } \\
\text { also be operative in great proportions and elastically rise and decrease way. }\end{array}$ \\
\hline Mobility C4 & $\begin{array}{l}\text { IoT computers, end consumers, and nodes of fog may be mobile. This versatility has to be managed by } \\
\text { Fog systems. }\end{array}$ \\
\hline Federation C5 & $\begin{array}{l}\text { Fog distributes large-scale deploys where each fog domain can belong to a separate provider as well as } \\
\text { numerous cloud components. The federation of these separate providers, which may host multiple } \\
\text { elements, is required to provide applications. }\end{array}$ \\
\hline Interoperability C6 & $\begin{array}{l}\text { The implementation of elements from different providers may be carried out as part of a federated } \\
\text { system. In provider level and design packages, fog computing must be fully compatible. }\end{array}$ \\
\hline
\end{tabular}

\subsubsection{Review on latest IoT smart services}

In the selected studies, Table 3 demonstrates the criteria and an overview of gaps that are not approached by such relevant studies.

Table 3. Main features and requirements in the fog computing environment for effective orchestration

\begin{tabular}{|c|c|c|c|c|c|c|c|c|}
\hline \multirow[t]{2}{*}{ Author } & \multirow[t]{2}{*}{ Application type } & \multirow[t]{2}{*}{ Evaluated parameter } & \multicolumn{6}{|c|}{ Criteria } \\
\hline & & & $\mathrm{C} 1$ & $\mathrm{C} 2$ & $\mathrm{C} 3$ & $\mathrm{C} 4$ & C5 & C6 \\
\hline José Santos et al. [31] & Smart City & Latency, bandwidth & $x$ & $\checkmark$ & $x$ & $\checkmark$ & $x$ & $x$ \\
\hline D. A. Chekired et al. [62] & Smart Grid & energy & $x$ & $\checkmark$ & $x$ & $x$ & $x$ & $x$ \\
\hline Minh-Quang Tran et al. [63] & Smart city & $\begin{array}{l}\text { latency, energy consumption, } \\
\text { and network load }\end{array}$ & $x$ & $x$ & $x$ & $x$ & $\checkmark$ & $x$ \\
\hline Eugene Siow et al. [64] & Smart city & $\begin{array}{l}\text { Latency, scalablity, resource- } \\
\text { constrained }\end{array}$ & $x$ & $x$ & $\checkmark$ & $x$ & $x$ & $\checkmark$ \\
\hline Marica Amadeo et al. [65] & Smart Campus & $\begin{array}{l}\text { Workload, Service } \\
\text { provisioning time }\end{array}$ & $\checkmark$ & $x$ & $x$ & $x$ & $x$ & $x$ \\
\hline Karima Velasquez et al. [13] & Smart city & $\begin{array}{l}\text { latency levels, location } \\
\text { awareness, and mobility }\end{array}$ & $\checkmark$ & $x$ & $\checkmark$ & $\checkmark$ & $x$ & $x$ \\
\hline Bilal Khalid Dar et al. [26] & $\begin{array}{l}\text { Smart Accident } \\
\text { Detection }\end{array}$ & $\begin{array}{l}\text { Network throughput, latency } \\
\text { and execution time }\end{array}$ & $x$ & $x$ & $x$ & $\checkmark$ & $\checkmark$ & $x$ \\
\hline Md. Muzakkir Hussain [66] & $\begin{array}{l}\text { Smart Management } \\
\text { Services }\end{array}$ & $\begin{array}{l}\text { Latency, power consumption, } \\
\text { and cost of architecture. }\end{array}$ & $x$ & $\checkmark$ & $x$ & $x$ & $x$ & $x$ \\
\hline Mohammad Aazam et al. [67] & Smart Healthcare & Network, latency & $x$ & $x$ & $x$ & $x$ & $\checkmark$ & $x$ \\
\hline $\begin{array}{l}\text { Mohammad Abdullah Al } \\
\text { Faruque } \text { et al. [68] }\end{array}$ & Smart Energy & $\cos t$ & $\checkmark$ & $\checkmark$ & $x$ & $x$ & $x$ & $x$ \\
\hline
\end{tabular}

\section{CONCLUSION}

A fog network distributed computing system is essential to reduce electricity consumption and to satisfy end users latency requirements, reduce the burden on the central data centre and provide localization services to process data in real-time. Fog computing is an advanced technology in our daily lives that needs to improve overall latency-sensitive applications such as disaster management, smart healthcare and smart

A review on orchestration distributed systems for IoT smart services in ... (Nor Syazwani Mohd Pakhrudin) 
transport networks. A distributed storage system must fulfil several criteria for use in the fog computing environment. This network will be reliable and latency-low in heterogeneous networks with hundreds of thousands of fog nodes. The implementation of the fog computing helps overcome that large data in IoT system. The usage of fog computing has decreases latency and storage compare to the cloud computing.

\section{ACKNOWLEDGEMENTS}

Authors would like to thank Research Management Centre (RMC), Universiti Teknologi MARA, UiTM Shah Alam, Selangor, Malaysia for the support funding grant in publishing this paper.

\section{REFERENCES}

[1] A. Yousefpour, et al., "All one needs to know about fog computing and related edge computing paradigms: A complete survey," Journal of Systems Architecture, vol. 98, pp. 289-330, 2019.

[2] V. Kochar and A. Sarkar, "Real time resource allocation on a dynamic two level symbiotic fog architecture," in 2016 Sixth International Symposium on Embedded Computing and System Design (ISED), 2016, pp. 49-55.

[3] S. Sarkar and S. Misra, "Theoretical modelling of fog computing: a green computing paradigm to support IoT applications," IET Networks, vol. 5, pp. 23-29, 2016.

[4] S. Svorobej, et al., "Simulating fog and edge computing scenarios: An overview and research challenges," Future Internet, vol. 11, p. 55, pp. 1-15, 2019.

[5] H. F. Atlam, et al., "Fog Computing and the Internet of Things: A Review," Big Data and Cognitive Computing, vol. 2, p. 10, pp. 1-18, 2018.

[6] P. Prakash, et al., "Fog computing: issues, challenges and future directions," International Journal of Electrical and Computer Engineering (IJECE), vol. 7, no. 6, pp. 3669-3673, 2017.

[7] M. Aljarah, et al., "Cooperative hierarchical based edge-computing approach for resources allocation of distributed mobile and IoT applications," International Journal of Electrical and Computer Engineering (IJECE), vol. 10, no. 1, pp. 296-307, 2020.

[8] Q. Qi and F. Tao, "A Smart Manufacturing Service System Based on Edge Computing, Fog Computing, and Cloud Computing," IEEE Access, vol. 7, pp. 86769-86777, 2019.

[9] P. Sareen, "Cloud Computing: Types, Architecture, Applications, Concerns, Virtualization and Role of IT Governance in Cloud," International Journal of Advanced Research in Computer Science and Software Engineering, vol. 3, no. 3, pp. 533-538, 2013.

[10] R. Shahzadi, et al., "Three tier fog networks: Enabling IoT/5G for latency sensitive applications," China Communications, vol. 16, pp. 1-11, 2019.

[11] A. V. Dastjerdi and R. Buyya, "Fog Computing: Helping the Internet of Things Realize Its Potential," Computer, vol. 49, pp. 112-116, 2016.

[12] B. McCormick, et al., "Distributed Orchestration in Cloud Data Centers," in 2019 IFIP/IEEE Symposium on Integrated Network and Service Management (IM), 2019, pp. 346-352.

[13] K. Velasquez, et al., "Service Orchestration in Fog Environments," in 2017 IEEE 5th International Conference on Future Internet of Things and Cloud (FiCloud), 2017, pp. 329-336.

[14] R. Jawabreh, et al., "FogNetwork Orchestration for Heterogeneous Networks," Proceedings of the 3rd International Conference on Future Networks and Distributed Systems, Paris, France, 2019.

[15] A. Swain and A. K. Ray, "QoS Management in WSN-MCN Convergence Network Using Priority Based Traffic Models," Indonesian Journal of Electrical Engineering and Computer Science, vol. 12, pp. 1159-1170, 2018.

[16] S. Kitanov and T. Janevski, "Hybrid environment service orchestration for fog computing," in Proceedings of the 51st International Scientific Conference on Information, Communication and Energy Systems and Technologies (ICEST 2016), 2016, pp. 91-94.

[17] Z. Wen, et al., "Fog Orchestration for Internet of Things Services," IEEE Internet Computing, vol. 21, pp. 16-24, 2017.

[18] V. Dahiya and S. Dalal, "Fog Computing: A Review on Integration of Cloud Computing and Internet of Things," in 2018 IEEE International Students' Conference on Electrical, Electronics and Computer Science (SCEECS), 2018, pp. 1-6.

[19] Z. Ma, et al., "High-Reliability and Low-Latency Wireless Communication for Internet of Things: Challenges, Fundamentals, and Enabling Technologies," IEEE Internet of Things Journal, vol. 6, pp. 7946-7970, 2019.

[20] "Latency Statistics, Verizon Enterprise," 2020. [Online]. Available: https://enterprise.verizon.com/terms/latency/

[21] J. Morley, et al., "Digitalisation, energy and data demand: The impact of Internet traffic on overall and peak electricity consumption," Energy Research \& Social Science, vol. 38, pp. 128-137, 2018.

[22] S. Carsten, et al., "Smart Services," Procedia-Social and Behavioral Sciences, vol. 238, pp. 192-198, 2018

[23] P. More, "Review of implementing fog computing," International Journal of Research in Engineering and Technology, vol. 4, pp. 335-338, 2015

[24] T. Francis and M. Madhiajagan, "A Comparison of Cloud Execution Mechanisms: Fog, Edge and Clone Cloud Computing," Proceeding of the Electrical Engineering Computer Science and Informatics, vol. 4, 2017, pp. 446-450. 
[25] H. Dubey, et al., "Fog Computing in Medical Internet-of-Things: Architecture, Implementation, and Applications," in S. U. Khan, et al. (eds), Handbook of Large-Scale Distributed Computing in Smart Healthcare, ed Cham, Springer International Publishing, pp. 281-321, 2017.

[26] B. K. Dar, et al., "Delay-Aware Accident Detection and Response System Using Fog Computing," IEEE Access, vol. 7, pp. 70975-70985, 2019.

[27] B. C. E. Barros, et al., "Fog Computing Model to Orchestrate the Consumption and Production of Energy in Microgrids," Sensors, vol. 19, 2019.

[28] N. H. B. Hisham, et al., "IoT Gaming System with Generating Power Energy," in 2019 IEEE 9th International Conference on System Engineering and Technology (ICSET), 2019, pp. 281-286.

[29] A. M. Al-Smadi, et al., "Emergent situations for smart cities: a survey," International Journal of Electrical \& Computer Engineering (IJECE), vol. 9, no. 6, pp. 4777-4787, 2019.

[30] A. Ksentini, et al., "IoT/cloud-enabled smart services: A review on QoS requirements in fog environment and a proposed approach based on priority classification technique," International Journal of Communication Systems, 2019.

[31] J. Santos, et al., "Fog Computing: Enabling the Management and Orchestration of Smart City Applications in 5G Networks," Entropy, vol. 20, p. 4, pp. 1-26, 2018.

[32] S. Tian, et al., "Smart healthcare: making medical care more intelligent," Global Health Journal, vol. 3, pp. 62-65, 2019.

[33] E. Badidi and K. Moumane, "Enhancing the Processing of Healthcare Data Streams using Fog Computing," in 2019 IEEE Symposium on Computers and Communications (ISCC), 2019, pp. 1113-1118.

[34] A. Solanas, et al., "Smart health: A context-aware health paradigm within smart cities," IEEE Communications Magazine, vol. 52, pp. 74-81, 2014.

[35] W. Tang, et al., "Fog-Enabled Smart Health: Toward Cooperative and Secure Healthcare Service Provision," IEEE Communications Magazine, vol. 57, pp. 42-48, 2019.

[36] R. A. Rahman, et al., "IoT-based personal health care monitoring device for diabetic patients," in 2017 IEEE Symposium on Computer Applications \& Industrial Electronics (ISCAIE), 2017, pp. 168-173.

[37] Z. Wen, et al., "Fog Orchestration for Internet of Things Services," IEEE Internet Computing, vol. 21, pp. 16-24, 2017.

[38] A. Verma, et al., "Large-scale cluster management at Google with Borg," Proceedings of the Tenth European Conference on Computer Systems, Bordeaux, France, 2015.

[39] S. Vaucher, et al., "SGX-Aware Container Orchestration for Heterogeneous Clusters," in 2018 IEEE 38th International Conference on Distributed Computing Systems (ICDCS) , 2018, pp. 730-741.

[40] B. Gasser, et al., "Is Eccentric Muscle Work Adequately Perceived? An Analysis with BORG-Scale," Deutsche Zeitschrift für Sportmedizin, vol. 69, pp. 344-350, 2018.

[41] Y. Vlasov, et al., "Prototyping tools for IoT systems based on virtualization techniques," in 2018 IEEE 9th International Conference on Dependable Systems, Services and Technologies (DESSERT), 2018, pp. 87-92.

[42] H. Chen and F. J. Lin, "Scalable IoT/M2M Platforms Based on Kubernetes-Enabled NFV MANO Architecture," in 2019 International Conference on Internet of Things (iThings) and IEEE Green Computing and Communications (GreenCom) and IEEE Cyber, Physical and Social Computing (CPSCom) and IEEE Smart Data (SmartData), 2019, pp. 1106-1111.

[43] P. Tsai, et al., "Distributed analytics in fog computing platforms using tensorflow and kubernetes," in 2017 19th Asia-Pacific Network Operations and Management Symposium (APNOMS), 2017, pp. 145-150.

[44] J. Santos, et al., "Towards Network-Aware Resource Provisioning in Kubernetes for Fog Computing Applications," in 2019 IEEE Conference on Network Softwarization (NetSoft), 2019, pp. 351-359.

[45] M. Aly, et al., "Kubernetes or OpenShift? Which Technology Best Suits Eclipse Hono IoT Deployments," in 2018 IEEE 11th Conference on Service-Oriented Computing and Applications (SOCA), 2018, pp. 113-120.

[46] Z. Zhong and R. Buyya, "A Cost-Efficient Container Orchestration Strategy in Kubernetes-Based Cloud Computing Infrastructures with Heterogeneous Resources," ACM Trans. Internet Technol., vol. 20, no. 2, pp. 1-24, 2020.

[47] D. Kim, et al., "TOSCA-Based and Federation-Aware Cloud Orchestration for Kubernetes Container Platform," Applied Sciences, vol. 9, pp. 1-13, 2019.

[48] C. Seiber, et al., "Tracking hazardous aerial plumes using IoT-enabled drone swarms," in 2018 IEEE 4th World Forum on Internet of Things (WF-IoT), 2018, pp. 377-382.

[49] Y. Yao, et al., "Energy Efficiency Characterization in Heterogeneous IoT System With UAV Swarms Based on Wireless Power Transfer," IEEE Access, vol. 8, pp. 967-979, 2020

[50] E. Nii, et al., "Dynamic Multiple Swarming for Mobile Sensing Cluster based on Swarm Intelligence," in 2019 IEEE 5th World Forum on Internet of Things (WF-IoT), 2019, pp. 961-966.

[51] W. Ding and W. Fang, "Target Tracking by Sequential Random Draft Particle Swarm Optimization Algorithm," in 2018 IEEE International Smart Cities Conference (ISC2), 2018, pp. 1-7.

[52] A. Kohlbacher, et al., "A low cost omnidirectional relative localization sensor for swarm applications," in 2018 IEEE 4th World Forum on Internet of Things (WF-IoT), 2018, pp. 694-699.

[53] M. A. Serhani, et al., "Self-adapting cloud services orchestration for fulfilling intensive sensory data-driven IoT workflows," Future Generation Computer Systems, vol. 108, pp. 583-597, 2020.

[54] P. Saha, et al., "Exploring the Fairness and Resource Distribution in an Apache Mesos Environment," in 2018 IEEE 11th International Conference on Cloud Computing (CLOUD), 2018, pp. 434-441. 
[55] P. Saha, et al., "Tromino: Demand and DRF Aware Multi-Tenant Queue Manager for Apache Mesos Cluster," in 2018 IEEE/ACM 11th International Conference on Utility and Cloud Computing (UCC), 2018, pp. 63-72.

[56] G. Rattihalli, "Exploring Potential for Resource Request Right-Sizing via Estimation and Container Migration in Apache Mesos," in 2018 IEEE/ACM International Conference on Utility and Cloud Computing Companion (UCC Companion), 2018, pp. 59-64.

[57] R. DelValle, et al., "Electron: Towards Efficient Resource Management on Heterogeneous Clusters with Apache Mesos," in 2017 IEEE 10th International Conference on Cloud Computing (CLOUD), 2017, pp. 262-269.

[58] S. López-Huguet, et al., "Vertical elasticity on Marathon and Chronos Mesos frameworks," Journal of Parallel and Distributed Computing, vol. 133, pp. 179-192, 2019.

[59] J. Herrera and G. Moltó, "Toward Bio-Inspired Auto-Scaling Algorithms: An Elasticity Approach for Container Orchestration Platforms," IEEE Access, vol. 8, pp. 52139-52150, 2020.

[60] Y. Wang, et al., "A Big Aurora Data Management Framework Toward Aurora Classification," in 2017 IEEE 15th Intl Conf on Dependable, Autonomic and Secure Computing, 15th Intl Conf on Pervasive Intelligence and Computing, 3rd Intl Conf on Big Data Intelligence and Computing and Cyber Science and Technology Congress(DASC/PiCom/DataCom/CyberSciTech), 2017, pp. 1284-1287.

[61] E. Truyen, et al., "A Comprehensive Feature Comparison Study of Open-Source Container Orchestration Frameworks," Applied Sciences, vol. 9, no. 5, p. 931, 2019.

[62] D. A. Chekired, et al., "Fog-Computing-Based Energy Storage in Smart Grid: A Cut-Off Priority Queuing Model for Plug-In Electrified Vehicle Charging," IEEE Transactions on Industrial Informatics, vol. 16, pp. 3470-3482, 2020.

[63] M. Q. Tran, et al., "Task Placement on Fog Computing Made Efficient for IoT Application Provision," Wireless Communications and Mobile Computing, vol. 2019, p. 6215454, 2019.

[64] E. Siow, et al., "Ewya: An Interoperable Fog Computing Infrastructure with RDF Stream Processing," in Internet Science, Cham, pp. 245-265, 2017.

[65] M. Amadeo, et al., "Fog Computing in IoT Smart Environments via Named Data Networking: A Study on Service Orchestration Mechanisms," Future Internet, vol. 11, p. 222, 2019.

[66] M. M. Hussain and M. M. S. Beg, "Fog Computing for Internet of Things (IoT)-Aided Smart Grid Architectures," Big Data and Cognitive Computing, vol. 3, no. 1, p. 8, 2019.

[67] M. Aazam, et al., "Health Fog for Smart Healthcare," IEEE Consumer Electronics Magazine, vol. 9, pp. 96-102, 2020.

[68] M. A. A. Faruque and K. Vatanparvar, "Energy Management-as-a-Service Over Fog Computing Platform," IEEE Internet of Things Journal, vol. 3, pp. 161-169, 2016.

\section{BIOGRAPHIES OF AUTHORS}

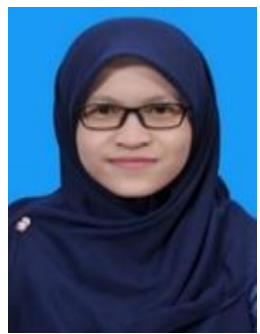

Nor Syazwani Mohd Pakhrudin is a PhD student in the Universiti Teknologi MARA (UiTM), Shah Alam, Selangor Malaysia in Electrical Engineering Program. She received her Master's degree in Telecommunication and Information Engineering, from Universiti Teknologi MARA (UiTM), Shah Alam, Selangor Malaysia under Faculty of Electrical Engineering in 2018. Previously, she obtained her first degree from Universiti Teknologi MARA (UiTM) Cawangan Pulau Pinang, with Honours, in Electrical and Electronic Engineering. She registered as a Graduate Engineer member with the Board of Engineers Malaysia (BEM) since 2018.

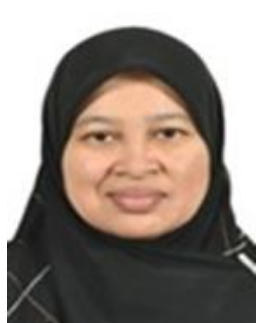

Murizah Kassim is currently working as Associate Professor at Faculty of Electrical Engineering, Universiti Teknologi MARA, Shah Alam, Selangor. She received her PhD in Electronic, Electrical and System Engineering in 2016 from the Faculty of Built Environment and Engineering, Universiti Kebangsaan Malaysia (UKM). She has published many indexed papers related to computer network, IoT, Web and Mobile development applications research. She experienced for 19 years in the technical team at the Centre for Integrated Information System, UiTM. She is also head of Enabling Internet of Things Technologies (ElIoTT) research group UiTM. She joined the academic since January 2009 and currently member of MBOT, IEEE, IET, IAENG and IACSIT organization.

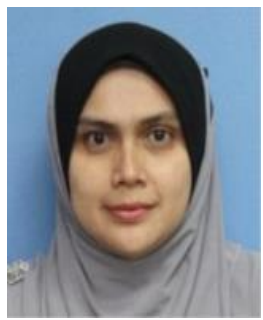

Azlina Idris is an Associate Professor at the Universiti Teknologi MARA (UiTM), Selangor, Malaysia. She obtained her $\mathrm{PhD}$ in wireless communication from University Malaya (UM), Malaysia. She has received the Master of Engineering in Electrical from Universiti Teknologi Malaysia (UTM). Previously, she obtained her first degree from Leeds Metropolitan University, United Kingdom with Honours, in Applied Computer. She is a member of Wireless Communication Technology (WiCOT) Research Interest Group (RIG) and her research interests include OFDM/OFDMA transmission, single and multiuser precoding, modulation, MIMO transmission techniques and receivers, channel coding, interference management and mitigation, and channel modeling (channel estimation). 